\title{
Conception d'outil par couplage des éléments de domaine et de contour
}

\author{
Dominique Bigota ${ }^{a}$ Hocine Kebir et Jean-Marc Roelandt \\ Laboratoire Roberval, UTC/CNRS, Université de Technologie de Compiègne, BP 20529, 60205 Compiègne Cedex, France
}

Reçu le 25 mai 2007, accepté le 24 janvier 2008

\begin{abstract}
Résumé - À l'heure actuelle, la conception des procédés de mise en forme s'appuie grandement sur la simulation numérique via l'utilisation de codes industriels. Ces simulations portent souvent sur la déformation des pièces et rarement sur la durée de vie des outils. Afin de prendre en compte les éventuelles modifications de l'outil au cours des cadences de mise en forme, nous développons un outil numérique dédié. Cet outil numérique permettra plus particulièrement l'identification des critères de fatigue de l'outil. Il sera alors possible de concevoir des formes optimales d'outils permettant d'augmenter leur durée de vie tout en assurant la qualité exigée sur les pièces ainsi fabriquées. Ce dernier utilise un couplage en contact avec frottement de la méthode des éléments-finis - pour modéliser la pièce - et de la méthode des éléments de frontière - pour modéliser l'outil. Dans cet article, nous traiterons un exemple de cisaillement.
\end{abstract}

Mots clés : Couplage éléments-finis / éléments frontières / pénalité / mise en forme / optimisation / durée de vie / critère de fatigue

\begin{abstract}
Conception of tool by coupling boundary elements and field element. Nowadays, the simulation of forming processes is based largely on numerical simulation via the industrial numerical codes. These simulations often relate on the deformation of the parts and seldom to the lifespan of the tools. However, to take into account the possible modifications of the tool during cycle of working, we develop dedicated numerical software. This one more particularly will allow the identification of the fatigue criteria of the tool. With the view to conceiving the optimal shapes of tool allowing increasing their lifespan while ensuring a quality required of the part thus manufactured. This latter uses contact coupling with friction finite element method - for modelling the part - and boundary element method - for modelling the tool. In this article, we treat an example of cutting process.
\end{abstract}

Key words: Coupling finite-elements / boundary elements / penalty / forming process / optimisation / lifespan / fatigue criteria

\section{Introduction}

Estimer la durée de vie des outils devient un axe de recherche prometteur. En effet, la connaissance de l'impact du nombre de passes sur la géométrie, l'usure, la fatigue d'un outil,... est peu développée numériquement. Pour prendre en compte l'usure de l'outil, une attention particulière doit être apportée au contact entre les surfaces de la pièce et de l'outil. Un outil numérique dédié a été conçu permettant l'identification des critères de fatigue de l'outil, dans le but de concevoir des formes d'outils optimales et donc d'augmenter leur durée de vie tout en assurant la qualité exigée sur les pièces ainsi

${ }^{a}$ Auteur pour correspondance :

dominique.bigot.2001@tremplin-utc.net fabriquées. En raison des particularités de chaque composant du procédé de fabrication, nous avons choisi un couplage éléments-finis/éléments-frontières, dont les caractéristiques sont (Fig. 1) :

- la modélisation de la pièce en éléments-finis : la pièce étant soumise à de grandes transformations géométriques, ainsi qu'à des non-linéarités matérielles,

- la modélisation de l'outil en éléments de frontière : l'outil est soumis à de petites déformations et discrétisé par des éléments de frontière,

- la mise en contact et le couplage élémentsfinis/éléments de frontière (le coefficient de frottement est pris égal à 0,15$)$.

L'algorithme de contact et la discrétisation des composants est repris de $[1,2]$ mais sera brièvemement 


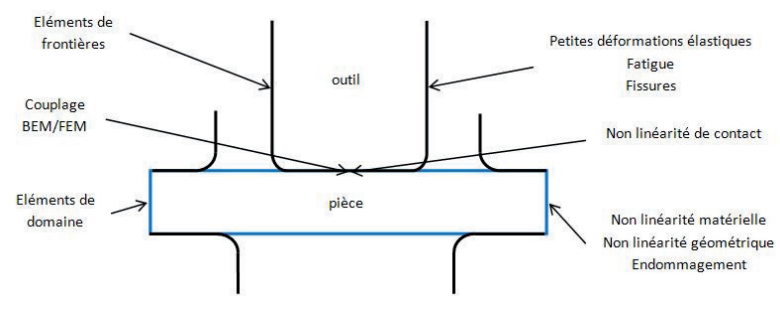

Fig. 1. Modélisation.

abordé dans la partie suivante. Le logiciel KSP, dédié aux procédés de mise en forme et développé au sein du Laboratoire Roberval, a été utilisé pour la modélisation du procédé de fabrication.

\section{Couplage en contact}

Dans cette section, l'algorithme de résolution des problèmes de contact $[1,2]$ est brièvement abordé. Il est basé sur une méthode de pénalité [3-7]. Un ajustement de paramètre de pénalité est developpé pour résoudre les problèmes numériques. Un ajustement automatique du pas de chargement est développé pour renforcer la robustesse de l'algorithme.

\subsection{Modélisation}

Le principe de la méthode des éléments de frontière $[8,9]$ consiste à transformer les équations intégrales de contour. En élasticité linéaire, l'étude d'un volume revient à étudier le comportement de sa surface. La discrétisation transforme l'équation intégrale de contour en un système d'équations linéaires :

$$
[A]\{u\}=[B]\{t\}
$$

où $\{u\}$ est le champ de déplacement et $\{t\}$ est le champ de tension.

La méthode des éléments-finis est une méthode utilisée dans le calcul des structures, particulièrement dans les domaines non-linéaires $[10,11]$. En partant du principe des puissances virtuelles et après discrétisation, la résolution $[12,13]$ peut se faire grâce à un processus itératif de Newton-Raphson :

$$
\left[K_{\mathrm{T}}\right]\{\triangle u\}=\left\{F_{\text {ext }}\right\}-\left\{F_{\text {int }}\right\}
$$

\subsection{Principe}

Le but est de corriger la pénétration de l'outil dans la pièce par le calcul des forces de contact (Fig. 2). Des calculs itératifs sont nécessaires à l'obtention de l'équilibre des deux systèmes, où

$x_{n}$ est la distance de pénétration;

$t_{1}$ est la distance de glissement;

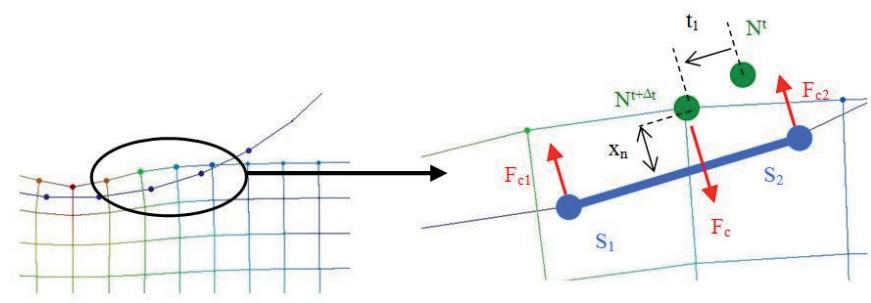

Fig. 2. Principe de résolution.

$F_{\mathrm{c}}$ est la force de contact associée au nœud de contact $N^{t+\Delta t}$

$F_{\mathrm{c} 1}$ et $F_{\mathrm{c} 2}$ sont les forces de contact associées au segment de contact $\left(S_{1} S_{2}\right)$.

L'algorithme [2] est basé sur le principe outil 《 maître »-pièce « esclave » (Fig. 3).

Pour chaque pas, la zone de contact est déterminée en identifiant le couple segment/nœud vérifiant $x_{n} \leq 0$, $\overrightarrow{S_{1} N} \cdot \overrightarrow{S_{1} S_{2}} \geq 0$ et $\overrightarrow{S_{2} N} \cdot \overrightarrow{S_{2} S_{1}} \geq 0$ (Fig. 2).

Pour chaque couple segment/nœud sont calculés

- la distance de pénétration $x_{n}$;

- la distance de glissement $t_{1}$;

- le paramètre normal de pénalité $k_{\mathrm{n}}$ - Pour le premier pas :

$$
k_{\mathrm{n}}=\frac{\sum_{i=1}^{n n c t} \mathrm{~K}_{i i}^{\mathrm{c}}}{n n c t}
$$

où $\left[\mathrm{K}^{\mathrm{c}}\right]$ est la matrice de rigidité associée aux nœuds de contact; nnct est le nombre de nœuds de contact.

- Pour les autres pas :

$$
k_{\mathrm{n}}=\frac{1}{2}\left(1+\frac{F_{\mathrm{c}}}{F_{\mathrm{c}}^{\max }}\right) k_{\text {moyen }}
$$

où $F_{\mathrm{c}}=\sqrt{\left(F_{\mathrm{c}}^{\mathrm{n}}\right)^{2}+\left(F_{\mathrm{c}}^{\mathrm{t}}\right)^{2}}$ force de contact pour le couple $\left(N_{i}, S_{j}\right), F_{\mathrm{c}}^{\max }=\max _{\left(N_{i}, S_{j}\right)}\left(F_{\mathrm{c}}\right) F_{\mathrm{c}}^{\mathrm{T}}=$ $\sum_{\left(N_{i}, S_{j}\right)} F_{\mathrm{c}} k_{\text {moyen }}=\frac{1}{n n c t} \frac{F_{\mathrm{c}}^{\mathrm{T}}}{\text { déplacement précédent }}$.

- le paramètre tangentiel de pénalité $k_{\mathrm{t}}$

$$
k_{\mathrm{t}}=\mu k_{\mathrm{n}}
$$

où $\mu$ est le coefficient de frottement.

- la force de contact normale $F_{\mathrm{c}}^{\mathrm{n}}$, corrigeant la pénétration des nouds dans l'outil :

$$
\begin{aligned}
F_{\mathrm{c}}^{\mathrm{n}}= & \sum_{i_{\text {pas }}=1}^{i_{\text {pas }}=n_{\text {pas }}-1}\left[\sum_{\text {iter }=1}^{n_{\text {iter }_{n}}}\left(\Delta F_{\mathrm{c}}^{\mathrm{n}}\right)_{i_{\text {pas }}}^{\text {iter }}\right. \\
& +\sum_{\text {iter }=1}^{\text {iter }=n_{\text {iter }_{i_{\text {pas }}}}}\left(\Delta F_{\mathrm{c}}^{\mathrm{n}}\right)_{n_{\text {pas }}}^{\text {iter }}
\end{aligned}
$$

où $n_{\text {pas }}$ est le nombre de pas atteint, 


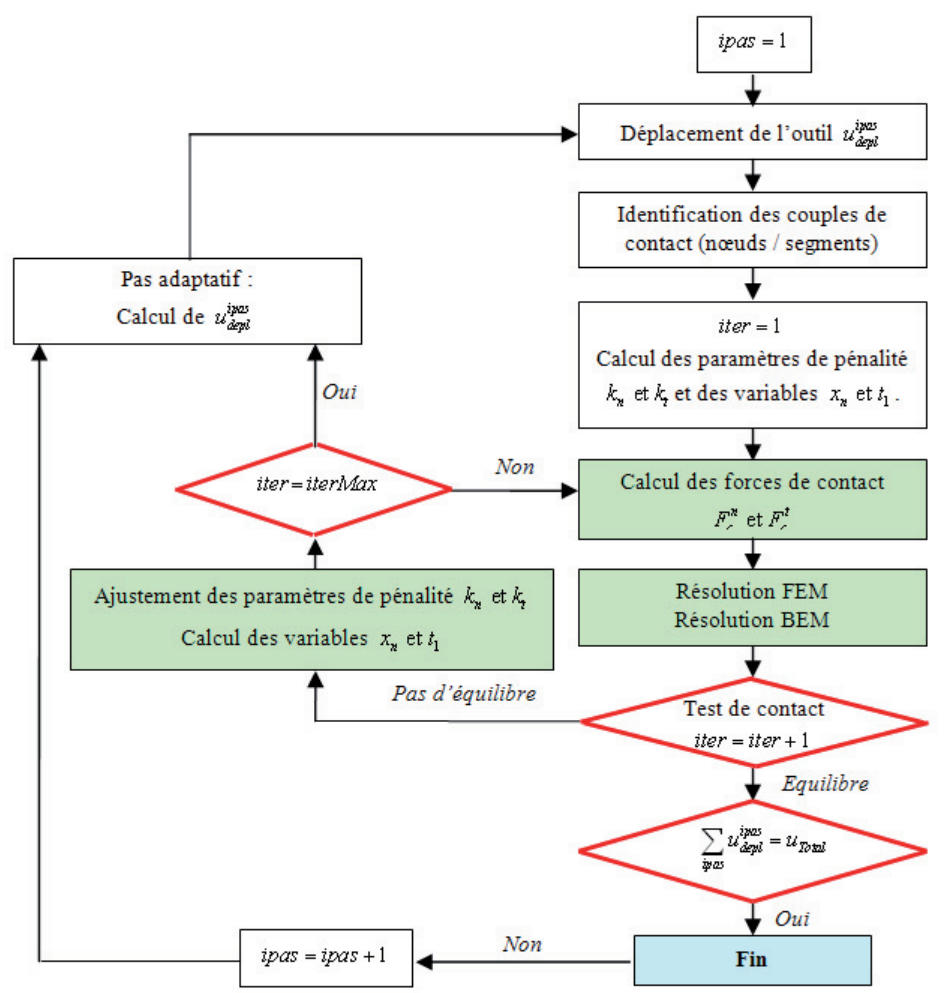

Fig. 3. Algorithme de contact.

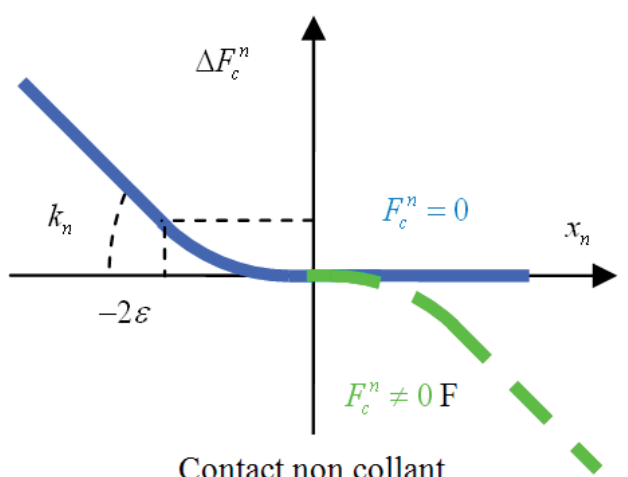

Fig. 4. Force de contact lissée.

$n_{\text {iter }_{\text {ipas }}}$ est le nombre d'itérations pour atteindre l'équilibre au pas $i_{\text {pas }}$,

$n_{\text {iter }_{n} \text { pas }}$ est le nombre d'itérations pour atteindre l'équilibre au pas $n_{\text {pas }}$,

$\Delta F_{\mathrm{c}}^{\mathrm{n}}=k_{\mathrm{n}} \Phi\left(x_{\mathrm{n}}\right)$ est l'incrément de force normale sur l'itération

$\Phi$ est une fonction régularisant la force de contact normale, en améliorant le statut de « contact/noncontact $»$. Elle est définie par (Fig. 4) :

- pénétration $\left(x_{\mathrm{n}} \leq 0\right)$ :

$$
\Phi\left(x_{\mathrm{n}}\right)=\left\{\begin{array}{l}
x_{\mathrm{n}} \text { si } x_{\mathrm{n}} \leq-2 \epsilon \\
\frac{\left(x_{\mathrm{n}}+\epsilon\right)^{2}}{4 \epsilon}-\frac{\left(x_{\mathrm{n}}+\epsilon\right)}{2}+\frac{\epsilon}{4} \\
\text { si }-2 \epsilon \leq x_{\mathrm{n}} \leq 0
\end{array}\right.
$$

- séparation $\left(x_{\mathrm{n}}>0\right)$ :

$$
\Phi\left(x_{\mathrm{n}}\right)=\left\{\begin{array}{l}
-x_{\mathrm{n}} \text { si } x_{\mathrm{n}} \geq 2 \epsilon \\
-\left(\frac{\left(x_{\mathrm{n}}+\epsilon\right)^{2}}{4 \epsilon}-\frac{\left(x_{\mathrm{n}}+\epsilon\right)}{2}+\frac{\epsilon}{4}\right)(8) \\
\text { si } 2 \epsilon \geq x_{\mathrm{n}} \geq 0
\end{array}\right.
$$

- la force de contact tangentielle $F_{\mathrm{c}}^{\mathrm{t}}$, prenant en compte le frottement, basée sur le modèle de frottement de Coulomb [14]:

$$
\begin{aligned}
F_{\mathrm{c}}^{\mathrm{t}}= & \sum_{i_{\text {pas }}=1}^{i_{\text {pas }}=n_{\text {pas }}-1}\left[\sum_{\text {iter }=1}^{n_{\text {iter }} n_{\text {pas }}}\left(\Delta F_{\mathrm{c}}^{\mathrm{t}}\right)_{i_{\text {pas }}}^{\text {iter }}\right] \\
& +\sum_{\text {iter }=1}^{\text {iter }=n_{\text {iter }} i_{\text {pas }}}\left(\Delta F_{\mathrm{c}}^{\mathrm{t}}\right)_{n_{\text {pas }}}^{\text {iter }}
\end{aligned}
$$

où

$$
\Delta F_{c}^{t}= \begin{cases}r_{\mathrm{t}} & \text { contact collant } \\ -\mu\left|\Delta F_{\mathrm{n}}^{\mathrm{c}}\right| \frac{t_{1}}{\left|t_{1}\right|} \text { et } k_{\mathrm{t}}=\mu k_{\mathrm{n}}\left|\frac{x_{\mathrm{n}}}{t_{1}}\right| \\ \text { si }\left|r_{\mathrm{t}}\right| \geq \mu\left|\Delta F_{\mathrm{n}}^{\mathrm{c}}\right| \quad \text { contact glissant }\end{cases}
$$
avec

$$
r_{\mathrm{t}}=-k_{\mathrm{t}} t_{1}
$$

\subsection{Résolution}

Les déplacements de la pièce et de l'outil sont évalués indépendamment par la résolution de deux systèmes distincts. 


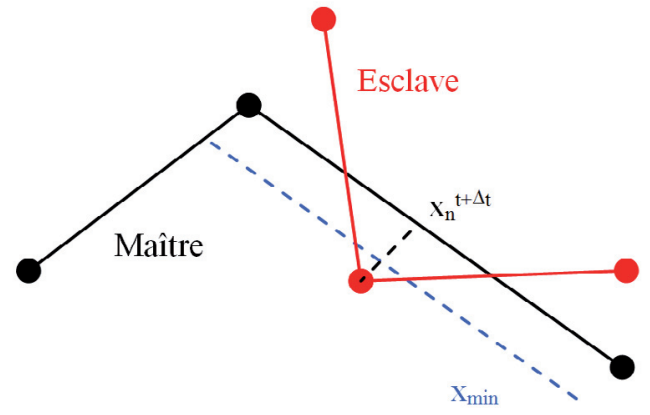

Fig. 5. Ajustement des paramètres de pénalité.

Pour la pièce, modélisée en élément-finis de domaine, le contact est pris en compte en ajoutant à (2) le vecteur des forces de contact $\left\{F_{\text {contact }}\right\}$ au second membre :

$$
\left[K_{\mathrm{T}}\right]\{\triangle u\}=\left\{F_{\text {ext }}\right\}-\left\{F_{\text {int }}\right\}+\left\{F_{\text {contact }}\right\}
$$

Pour l'outil, modélisé en éléments de frontière, le contact est pris en compte en ajoutant à (1) le vecteur des tensions de contact $\left\{t_{\text {contact }}\right\}$ au vecteur des tensions $\{t\}$ :

$$
[A]\{u\}=[B]\left(\{t\}+\left\{t_{\text {contact }}\right\}\right)
$$

L'outil et la pièce sont considérés en contact quand l'équilibre entre les deux systèmes est atteint.

La détermination de l'équilibre des deux systèmes est basée sur trois tests :

- Le premier test verifie l'équilibre entre les surfaces de contact. Si la distance de pénétration est trop petite $x_{\mathrm{n}}^{t+\Delta t}<\epsilon_{1}$, l'équilibre est atteint.

- Le second test prend en compte les nœuds qui sortent de la zone de contact.

- Le nœud ne pénètre plus l'outil :

$$
x_{\mathrm{n}}^{t+\Delta t}>0
$$

- La variation du déplacement est négligeable :

$$
\frac{\left|x_{\mathrm{n}}^{t+\Delta t}-x_{\mathrm{n}}^{t}\right|}{\left|x_{\mathrm{n}}^{t}\right|}<\epsilon_{2}
$$

- La force de contact est presque nulle :

$$
\left|F_{\mathrm{c}}\right|=\sqrt{\left(F_{\mathrm{c}}^{\mathrm{n}}\right)^{2}+\left(F_{\mathrm{c}}^{\mathrm{t}}\right)^{2}}<\epsilon_{3}
$$

- Le troisième test vérifie si les nœuds glissent sur la surface.

$$
\frac{\left|t_{1}^{t+\Delta t}-t_{1}^{t}\right|}{\left|t_{1}^{t}\right|}<\epsilon_{3}
$$

Lorsque le test d'équilibre est satisfait, l'algorithme passe au pas suivant, au contraire, les paramètres de pénalité normal et tangentiel sont ajustés pour l'itération précédente [15].

Le paramètre de pénalité normal $k_{\mathrm{n}}$ est ajusté grâce à la fonction $\Psi_{\mathrm{n}}$ (Fig. 5) :

$$
k_{\mathrm{n}} \longrightarrow \Psi_{\mathrm{n}}\left(x_{\mathrm{n}}^{t+\Delta t}, x_{\min }\right) k_{\mathrm{n}}
$$

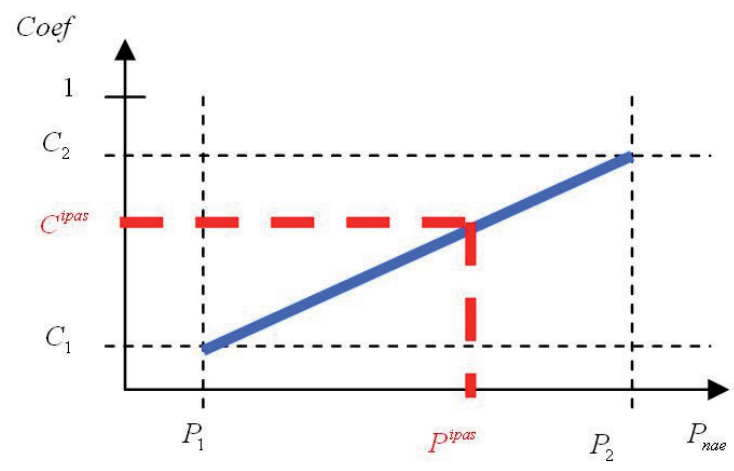

Fig. 6. Pas adaptatif.

où

$\Psi_{\mathrm{n}}\left(x_{\mathrm{n}}^{t+\Delta t}, x_{\min }\right)=\left\{\begin{array}{ccc}\frac{\left|x_{\mathrm{n}}^{t+\Delta t}\right|}{x_{\text {min }}} & \text { si } \frac{\left|x_{\mathrm{n}}^{t+\Delta t}-x_{\mathrm{n}}^{t}\right|}{\left|x_{\mathrm{n}}^{t}\right|}<\epsilon_{4} \\ \frac{x_{\mathrm{n}}^{t}}{x_{\mathrm{n}}^{t+\Delta t}-x_{\mathrm{n}}^{t}} \mid & \text { si } \frac{\left|x_{\mathrm{n}}^{t+\Delta t}-x_{\mathrm{n}}^{t}\right|}{\left|x_{\mathrm{n}}^{t}\right|}>\epsilon_{5} \\ 1 & \text { sinon }\end{array}\right.$

Le paramètre de pénalité tangentiel $k_{\mathrm{t}}$ est ajusté grâce à la fonction $\Psi_{\mathrm{t}}$ :

$$
k_{\mathrm{t}} \longrightarrow \Psi_{\mathrm{t}}\left(t_{1}^{t}, t_{1}^{t+\Delta t}\right) k_{\mathrm{t}}
$$

où

$$
\Psi_{t}\left(t_{1}^{t}, t_{1}^{t+\Delta t}\right)=\left\{\begin{array}{cc}
1 & \text { si }\left|t_{1}^{t+\Delta t}\right|<\epsilon_{6} \\
\text { et } t_{1}^{t} t_{1}^{t+\Delta t} \geq 0 & \text { sinon }
\end{array}\right.
$$

L'ajustement des paramètres de pénalité sont très important. Un bon ajustement conditionne la rapidité de l'algorithme de contact, mais aussi sa robustesse. Au contraire, un mauvais ajustement aura des effets sur la rapidité de convergence de l'algorithme, lequel convergera plus lentement voire pas du tout.

La méthode, utilisée pour ajuster le pas de déplacement de l'outil, consiste à calculer un coefficient $C^{i_{\text {pas }}}$ tel que :

$$
u_{\mathrm{depl}}^{i_{\mathrm{pas}}} \longrightarrow C^{i_{\mathrm{pas}}} u_{\mathrm{depl}}^{i_{\mathrm{pas}}}
$$

Si les nœuds de contact ne sont pas validés par les tests d'équilibre, le coefficient diminue le pas de déplacement. Le calcul du coefficient prend en compte le pourcentage de nœuds de contact respectant les conditions de contact (Fig. 6).

Si les nouds de contact sont validés par les tests d'équilibre, le coefficient augmente le pas de déplacement. Le calcul du coefficient prend en compte le nombre d'itérations nécessaire à l'équilibre du pas précédent (Fig. 7).

Après avoir rappelé la méthode de contact, les parties suivantes vont traiter du problème d'optimisation de l'outil. 


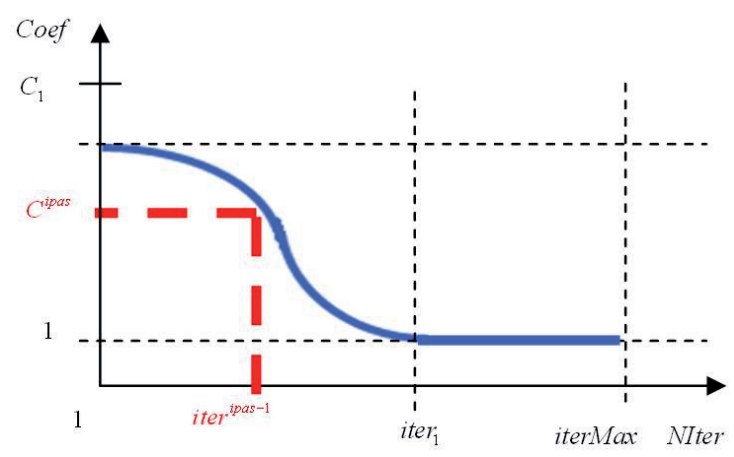

Fig. 7. Pas adaptatif.

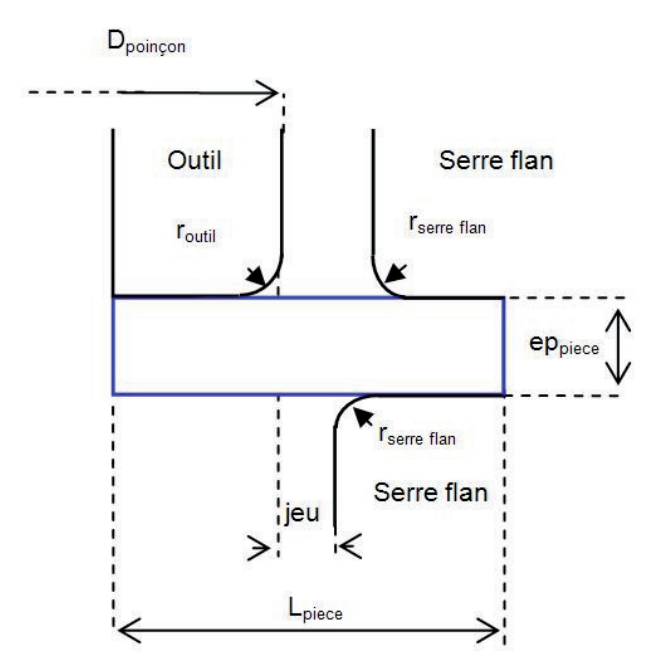

Fig. 8. Procédés de découpe.

\section{Description du problème}

La réalisation de pièces, grâce aux procédés de mise en forme, exige la conception d'outils spécifiques. Cet outil doit :

- assurer une qualité de conception à la pièce ;

- maximiser la durée de vie de l'outil.

Le respect de ces deux critères définit le problème à résoudre pour concevoir des formes d'outils optimales.

Le procédés de mise en forme de la découpe d'une pièce par un outil est étudiée. Les caractéristiques de l'essai sont les suivantes (Fig. 8) :

La pièce est modélisée en éléments-finis. Ses caractéristiques et dimensions sont les suivantes :

$$
\left\{\begin{array}{l}
e p_{\text {pièce }}=1,5 \mathrm{~mm} \\
L_{\text {pièce }}=6 \mathrm{~mm} \\
E_{\text {pièce }}=70000 \mathrm{MPa} \\
v_{\text {pièce }}=0,3 \\
\sigma_{0}=243 \mathrm{MPa} \\
H_{\mathrm{p}}=2240 \mathrm{MPa}
\end{array}\right.
$$

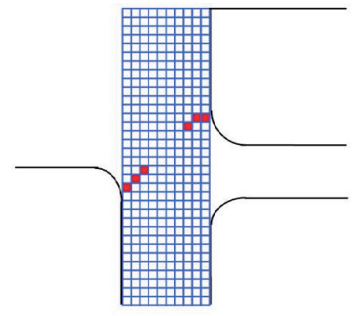

Fig. 9. Éléments endommagés.

L'outil est modélisé en éléments de frontière. Ses caractéristiques et dimensions sont les suivantes :

$$
\left\{\begin{array}{l}
D_{\text {poinçon }}=6 \mathrm{~mm} \\
r_{\text {outil }}=\text { variable à optimiser } \\
j e u=\text { variable à optimiser } \\
E_{\text {outil }}=210000 \mathrm{MPa} \\
v_{\text {outil }}=0,3
\end{array}\right.
$$

Les serre-flans sont modélisés en éléments de frontière, mais rigides :

$$
r_{\text {serre flan }}=r_{\text {outil }}
$$

Le coefficient de frottement entre les différents composants est :

$$
\mu=0,15
$$

Pour prendre en compte la rupture de la pièce, la variable d'endommagement de Rice et Tracey est utilisée [16] :

$$
\frac{\mathrm{d} R}{R} \simeq 0,283 \exp \left(\frac{2}{3} \frac{\sigma_{\mathrm{h}}}{\sigma_{\mathrm{eq}}}\right) \mathrm{d} \varepsilon_{\mathrm{e}}^{\mathrm{p}}
$$

La pièce a rompu quand une série d'éléments est endommagée (Fig. 9).

L'intégration du critère de Rice et Tracey donne :

$$
\log \left(\frac{R}{R_{0}}\right)=\int_{\epsilon_{0}^{\mathrm{p}}}^{\epsilon_{\mathrm{f}}^{\mathrm{p}}} 0,283 \exp \left(\frac{2}{3} \frac{\sigma_{\mathrm{h}}}{\sigma_{\mathrm{eq}}}\right) \mathrm{d} \epsilon_{\mathrm{e}}^{\mathrm{p}}
$$

où :

$R_{0}$ est le rayon initial de la cavité;

$\sigma_{\mathrm{h}}$ est la contrainte hydrostatique;

$\sigma_{\text {eq }}$ est la contrainte équivalente;

$d \epsilon_{\mathrm{e}}^{\mathrm{p}}$ est l'incrément de déformation plastique équivalent.

Un élément est considéré comme endommagé lorsque la cavité augmente de plus de dix pour cent (Fig. 10).

Dans cet exemple de découpe, les variables à optimiser sont le jeu et le rayon de l'outil $r_{\text {outil }}$. L'objectif est de minimiser la hauteur de bavure en assurant une durée de vie minimale à l'outil.

Aprés la rupture, la hauteur de bavure $h_{\mathrm{b}}$ détermine la qualité de la pièce fabriquée (Fig. 11). De ce fait, plus la hauteur de bavure est petite, meilleure est la qualité de la pièce. 


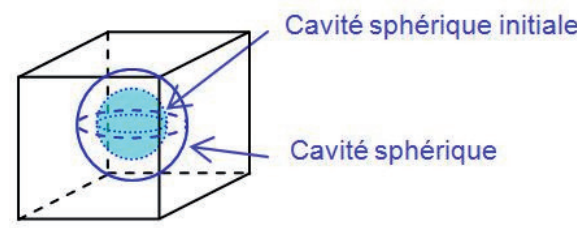

Fig. 10. Croissance de la cavité sphérique.
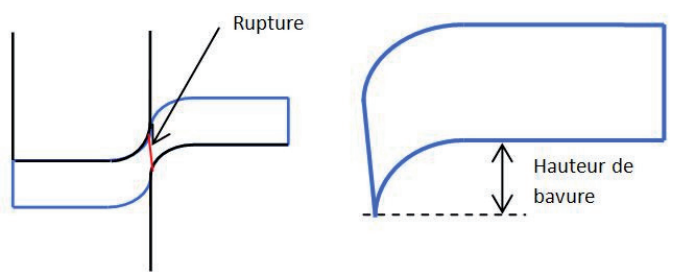

Fig. 11. Hauteur de bavure $h_{\mathrm{b}}$.

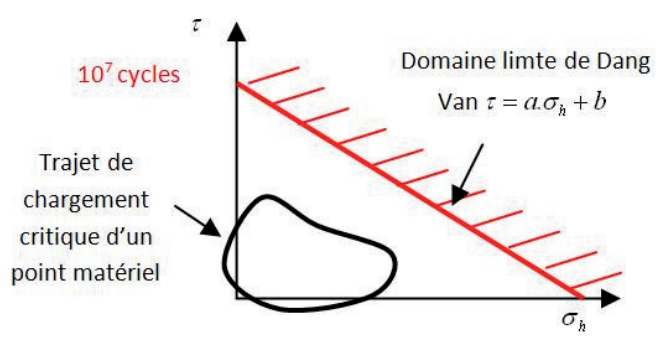

Fig. 12. Domaine admissible de Dang Van.

La fonction objective à minimiser s'écrit :

$$
F\left(r_{\text {outil }}, j e u\right)=h_{\mathrm{b}}
$$

La fatigue de l'outil est déterminée grâce au critère de Dang Van [17-19]. La durée de vie de l'outil est déterminée traçant le trajet de chargement d'un point matériel dans le repère $\left(\tau, \sigma_{\mathrm{h}}\right)$ et le domaine admissible de Dang Van (Fig. 12).

La fonction limitation est basée sur la distance entre le trajet de chargement et la droite limite de Dang Van. Cette fonction s'écrit :

$$
G\left(r_{\text {outil }}, j e u\right)=\min \left(\frac{\tau-a \sigma_{\mathrm{h}}-b}{\sqrt{a^{2}+1}}\right)
$$

Les coefficients $a$ et $b$ sont les constantes associées au matériau utilisées dans le critère de Dang Van. Ces constantes ont prises telles que $a=0,35$ et $b=1500 \mathrm{MPa}$. La fonction limitation s'écrit :

$$
G\left(r_{\text {outil }}, j e u\right) \leq 0
$$

En résumé, avec (24) et (25), le système à optimiser est le suivant :

$$
\left\{\begin{array}{l}
\min _{r_{\text {outil }}}, j e u\left(F\left(r_{\text {outil }}, j e u\right)\right) \\
G\left(r_{\text {outil }}, j e u\right) \leq 0
\end{array}\right.
$$

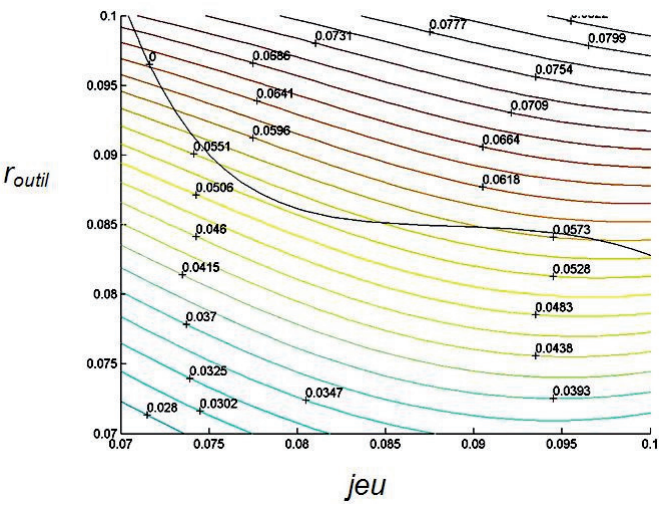

Fig. 14. Solution $\left(r_{\text {outil }}^{*}, j e u^{*}\right)$.

\section{Méthode d'optimisation et validation}

L'algorithme de résolution (Fig. 13) permettant de résoudre le système (26) est basé sur une méthode de surfaces de réponse.

Pour chaque fonction $F$ et $G$, les fonctions lissées $\tilde{F}$ et $\tilde{G}$ sont de forme polynomiale bicubique :

$$
\left\{\begin{array}{l}
\tilde{F}\left(r_{\text {outil }}, j e u\right)=\sum_{i=0}^{3} \sum_{j=0}^{3} a_{i j} r_{\text {outil }} j e u^{j} \\
\tilde{G}\left(r_{\text {outil }}, j e u\right)=\sum_{i=0}^{3} \sum_{j=0}^{3} b_{i j} r_{\text {outil }} j e u^{j}
\end{array}\right.
$$

Grâce à la modélisation numérique du procédé, $F_{\mathrm{n}}=$ $F\left(\left(r_{\text {outil }}\right)_{\mathrm{n}}, j e u_{\mathrm{n}}\right)$ et $G_{\mathrm{n}}=G\left(\left(r_{\text {outil }}\right)_{\mathrm{n}}, j e u_{\mathrm{n}}\right)$ sont calculés. Le nuage de points est constitué de vingt-cinq points balayant le domaine de définition.

Après avoir déterminé les surfaces de réponse $\tilde{F}$ et $\tilde{G}$ (27), le système (26) peut être résolu

$$
\left\{\begin{array}{l}
\min _{r_{\text {outil }}, \text { jeu }}\left(\tilde{F}\left(r_{\text {outil }}, j e u\right)\right) \\
\tilde{G}\left(r_{\text {outil }}, \text { jeu }\right) \leq 0
\end{array}\right.
$$

par une méthode numérique séquentielle de programmation quadratique (SQP) utilisé dans Matlab. Une solution $\left(r_{\text {outil }}^{*}, j e u^{*}\right)$ est trouvée et, grâce à la modélisation numérique du procédé, $F\left(r_{\text {outil }}^{*}, j e u^{*}\right)$ et $G\left(r_{\text {outil }}^{*}, j e u^{*}\right)$ sont calculés.

Deux cas sont considérés :

- Si $\left|G\left(r_{\text {outil }}^{*}, j e u^{*}\right)\right| \leq \epsilon$, alors $\left(r_{\text {outil }}^{*}, j e u^{*}\right)$ est la solution optimale du système 26 .

- Sinon, les valeurs $\left(r_{\text {outil }}^{*}, j e u^{*}, F\left(r_{\text {outil }}^{*}, j e u^{*}\right)\right)$ et $\left(r_{\text {outil }}^{*}, j e u^{*}, G\left(r_{\text {outil }}^{*}, j e u^{*}\right)\right)$ sont ajoutées aux nuages de points.

Après sept itérations de l'algorithme d'optimisation, la condition d'arrêt est vérifiée. La recherche de l'optimum donne :

$$
\begin{aligned}
& \left\{\begin{array} { l } 
{ r _ { \text { outil } } = 0 , 0 8 4 5 1 \mathrm { mm } } \\
{ \text { jeu } = 0 , 0 7 8 5 3 \times e p _ { \text { pièce } } }
\end{array} \left\{\begin{array}{l}
\tilde{F}=0,05342 \\
\tilde{G}=-6,913 \times 10^{-7}
\end{array}\right.\right. \\
& \left\{\begin{array}{l}
F=0,05087 \\
G=4,542
\end{array}\right.
\end{aligned}
$$




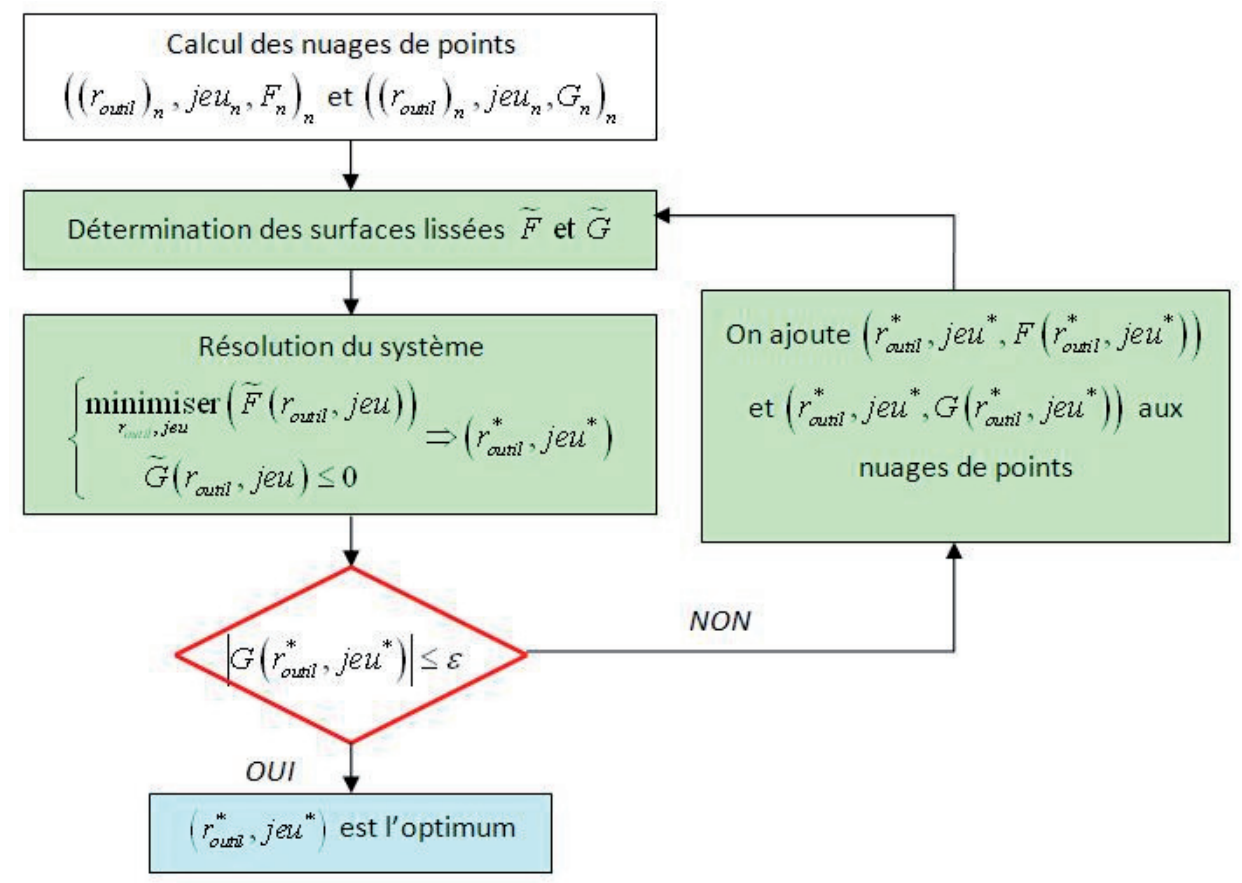

Fig. 13. Algorithme de recherche d'optimum.

La solution est déterminée à partir des surfaces de réponses. Ces dernières n'étant qu'une approximation du comportement réel, la solution trouvée est une approximation de l'optimum pour le problème de référence.

\section{Conclusions et perspectives}

Nous avons mis en place un algorithme d'optimisation permettant de maximiser la durée de vie d'un outil de découpe. La modélisation utilisée est basée sur le couplage éléments-finis/éléments de frontière permettant la simulation des procédés de mise en forme. Notre algorithme a donné des résultats prometteurs (convergence après sept simulations).

Par la suite, diverses perspectives intéressantes peuvent être suivies, comme :

- augmenter le nombre de variables à optimiser, par exemple en considérant l'angle paramétrant la pointe de l'outil;

- étendre le modèle aux cas axisymétrique et tridimensionnel;

- inclure un endommagement couplé (de type Guron ou Lemaitre);

- effectuer des corrélations calculs/essais à partir de données fournies par des partenaires industriels;

- prendre en compte l'usure de l'outil par écaillage;

- prendre en compte le retrait de l'outil après perçage ou découpe des tôles, qui provoque des frottements très dommageables pour l'outil ;

- prendre en compte les non-linéarités matérielles développées en surface de l'outil dues aux aspects mécaniques et thermiques.

\section{Références}

[1] D. Bigot, H. Kebir, J.M. Roelandt, Simulation de procédés de mise en forme couplant éléments finis de domaine et de frontière, $7^{\mathrm{e}}$ colloque national en calcul des structures, Giens, mai 2005

[2] D. Bigot, H. Kebir, J.M. Roelandt, Simulation of forming processes, with friction, coupling finite elements and boundary elements, IDMME06, Grenoble, May 17192006

[3] D. Peric, D.R.J. Oden, Computational model for the 3D contact problems with friction based on the penalty method, Int. J. Num. Meth. Engng. 35 (1992) 1289-1309

[4] I. Hunek, On a penalty formulation for contact-impact problems, Comp. Struct. 48 (1993) 193-203

[5] N. Kikuchi, J.T. Oden, Contact problems in elastostatics, Finite elements 5, ed. J.T. Oden, G.F. Carey, PrenticeHall, Englewood cliffs, NJ, 1984

[6] D. Chamoret, A. Rassineux, P. Villon et J.-M. Bergheau, Régularisation d'une surface de contact par approximation diffuse, Revue Européenne des Éléments Finis 11 (2002) 431-445

[7] D. Chamoret, P. Saillard, A. Rassineux, J.-M. Bergheau, New smoothing procedures in contact mechanics, J. Comp. Appl. Math. 168 (2004) 107-116

[8] M. Bonnet, Équations intégrales et éléments de frontière - Applications en mécanique des solides et des fluides, Éditions Eyrolles, 1995

[9] C.A. Brebbia, J. Dominguez, Boundary elements: an introductory course, Mc Graw-Hill, first edition, 1989

[10] L.E. Malvern, Introduction to the Mechanics of Continuum Medium, Prentice Hall, 1969

[11] J. Salençon, Mécanique des milieux continus, concepts généraux, École Polytechnique, Ellipse, 1988 
[12] P.V. Marcal, I.P. King, Elastic plastic analysis of two dimensional stress systems by the finite element method, Num. Meth. Ind. Form. Swansea 9 (1967)

[13] J.C. Nagtegaal, L.M. Taylor, Comparaison of implicit and explicit finite element methods for analysis of sheet forming problems, Proc. 1st Int. Conf. NUMISHEET'91, Zurich, Suisse, 1991, pp. 705-725

[14] C.A. Coulomb, Mémoires de Mathématique et de Physique de l'Académie Royale des Sciences, 1785

[15] S.H. Lee, Rudimentary consideration for adaptive gap/friction element based on the penalty method, Comp. Struct. 47 (1993) 1243-1056

[16] J.R. Rice, D.M. Tracey, On the ductile enlargement of voids in triaxial stress field, J. Mech. Phys. Solids 17 (1969) 201-217
[17] K. Dang Van, B. Griveau, O. Message, On a new multiaxial fatigue limit criterion: Theory and application, biaxial and multiaxial fatigue, ed. M.W. Brown, K. Miller, EGF Publication 3 (1982) 479-496

[18] K. Dang Van, Macro Micro approach in high cycle multiaxial fatigue, advances in multiaxial fatigue, ASTM STP 1991, ed. D.L. McDowell, R. Ellis, Am. Soc. testing and materials, Philadelphia, 1993, pp. 120-130

[19] K. Dang Van, Introduction to fatigue analysis in mechanical design by the multiscale approach, high cycle metal fatigue in the context of mechanical design, ed. K. Dang Van, I. Papadoupoulos, CISM Courses and lectures, No. 392, Springer-Verglag, 1999, pp. 57-88 